\title{
SECOND ORDER SLIDING MODE CONTROL OF COUPLED TANKS
}

\author{
Mohammad Khalid Khan ${ }^{*, 1}$ Sarah K Spurgeon*
}

\author{
* Control $\& 3$ Instrumentation Research Group \\ Department of Engineering \\ University of Leicester, LE1 7RH, U.K. \\ e-mail: eon@le.ac.uk \\ Tel.: +44-116-252-2531, Fax.: +44-116-252-2619
}

\begin{abstract}
A second order sliding mode control algorithm is presented for robust control of the liquid level in a pair of interconnected tanks. The controller is implemented on a laboratory rig. The implementation results show robustness to parameter variations such as tank area, the admittance coefficients of various pipes, leakage in the tanks and uncertainty in the pump dynamics. The potential of second order sliding modes for multivariable control is demonstrated.

Copyright $^{\circledR}$ 2005 IFAC.
\end{abstract}

Keywords: Second order sliding modes, MIMO liquid level control

\section{INTRODUCTION}

Sliding Mode Control (SMC) is known to be a robust control method appropriate for uncertain systems. High robustness is maintained against various kinds of uncertainties such as external disturbances and measurement error (Utkin (1992), Edwards and Spurgeon (1998)). It is also straightforward to implement the resulting algorithms. In such traditional sliding mode control, or First Order Sliding Mode (FOSM) controller design, an appropriate sliding variable, $s(t)$, is selected such that it has relative degree one with respect to the control. This selection of sliding variable must be made to ensure that the dynamics of the system in the sliding mode, i.e. when $s=0$, are desirable. The control then acts on the first derivative (with respect to time) of the sliding variable $(\dot{s})$ to keep the system trajectories in the sliding set $s=0$. Essentially, the discontinuous control signal acts on the first derivative of $s$ to ensure the system trajectories are always directed towards $s(t)=0$.

\footnotetext{
1 supported by EPSRC Grant Reference GR/M94021
}

Higher Order Sliding Modes (HOSM) are the generalisation of FOSM. In HOSM control, a control is sought which acts on higher derivatives of the sliding variable. For example, the case of second order sliding modes corresponds to the control acting on the second derivative of the sliding variable, namely $\ddot{s}$, and the sliding set is defined as $s=\dot{s}=0$. Higher order sliding mode control has the advantage, when compared to FOSM control, that it removes chattering effects, providing a smooth or at least piece-wise smooth control, and provides better performance with respect to switching delays in the control implementation.

This paper considers the application of second order sliding mode control techniques for MIMO liquid level control. A second order sliding mode control has been proposed for MIMO systems by Chang (1990) where a dynamics is imposed on the sliding variable but the resulting controller requires the derivative of the sliding variable for implementation. Bartolini et. al (2000) extended a SISO algorithm for a class of nonlinear MIMO systems. However, the algorithm still requires sin- 
gular values of the sliding variable (maxima, minima or flex points) to be detected online. The method proposed in this paper uses a second order sliding mode control which does not require the derivative of the sliding surface vector to be available. Further, the sliding surface may or may not be control dependent. In either case, the method always produces a dynamic controller, contrary to the work by Lu and Spurgeon (1998) and SiraRamirez (1993). The proposed method combines elements from Sira-Ramirez (1993) and $\mathrm{Lu}$ and Spurgeon (1998) together with Chang (1990) and Bartolini et. al (2000) and is applicable to a wide class of systems which are not necessarily affine in the control. Section 2 presents a brief review of the second order sliding mode control design approach for single input single output (SISO) systems. The methodology is extended and applied to the multiple input multiple output (MIMO) situation in Section 3. Section 4 describes the twin tank process of interest. The results of controller implementation on an experimental rig are presented in Section 5. The experimental results validate the proposed theoretical approach.

\section{A SISO PROBLEM FORMULATION}

Consider an uncertain SISO nonlinear system

$$
\dot{x}=\phi(t, x(t))+\gamma(t, x(t)) u(t) ;
$$

which is affine in the control $u$ and where $x \in X \subset$ $\Re^{n}$ is a state vector, $u \in U \subset \Re$ is a bounded input and $t$ is the independent time variable. Select a sliding surface

$$
s=S(t, x)
$$

such that by zeroing it, the control objective is achieved. Further assume that the sliding surface, $s$, has relative degree two with respect to the control input i.e. $\frac{\partial}{\partial x} S(t, x) \gamma(t, x)=0$. Thus the system dynamics can be written in the following form

$$
\ddot{s}=f(t, s, \dot{s}, x)+g(t, s, \dot{s}, x) u
$$

The dynamics in equation (2) are assumed to satisfy the following bounding conditions

$$
\begin{aligned}
& 0<G_{\min } \leq g(t, s, \dot{s}) \leq G_{\max } \\
& |f(t, s, \dot{s})| \leq F ; \quad|s| \leq s_{0}
\end{aligned}
$$

where $G_{\min }, G_{\max }, s_{0}$ and $F$ are some positive constants. Given the physical limits of most practical engineering systems, the assumed bounding conditions are not unduly restrictive.

In Khan et al. (2003), a 2-sliding control algorithm is applied to stabilise the dynamics (2) without the knowledge of the derivative of the sliding variable $(\dot{s})$. The algorithm is given as follows

$$
u(t)=-\lambda \operatorname{sign}(s)+u_{2}(t)
$$

$$
\dot{u}_{2}= \begin{cases}-k u, & |u|>u_{0} \\ -W \operatorname{sign}(s), & |u| \leq u_{0}\end{cases}
$$

The corresponding sufficient conditions for finite time convergence are

$$
u_{0}>\frac{F}{G_{\min }} ; \quad \lambda>u_{0} ; \quad k, W>0
$$

\section{A MIMO PROBLEM FORMULATION}

Consider a locally observable general MIMO nonlinear system in state space form: $\dot{x}=\psi(x, u, t)$ and the output $y=h(x, u, t)$, where $x \in \Re^{n}$, $u \in \Re^{m}, y \in \Re^{p}$ and $\psi: \Re^{n} \times \Re^{m} \times \Re_{+} \rightarrow \Re^{n}$ and $h: \Re^{n} \times \Re^{m} \times \Re_{+} \rightarrow \Re^{p}$ are smooth vector functions. The following locally equivalent differential I-O form exists

$$
y_{i}^{\left(n_{i}\right)}=\phi_{i}(\hat{y}, \hat{u}, t), \quad i=1, \cdots, p .
$$

where $\hat{u}=\left(u, \cdots, u_{j}^{\left(\beta_{j}\right)}\right) ; j=1, \cdots, m$ and $\hat{y}=$ $\left(y, \cdots, y_{i}^{\left(n_{i}-1\right)}\right)$ with $\sum_{i}^{p} n_{i}=n$. This representation is the same as the Local Generalized Controller Canonical Form (LGCCF) in Fliess (1990). A differential I-O system is called proper if

(a) $p=m$,

(b) all $\phi_{i}, i=1, \cdots, m$ are $C^{1}$ functions,

(c) the following regularity condition is satisfied

$$
\operatorname{det}\left[\frac{\partial\left(\phi_{1}, \ldots ., \phi_{m}\right)}{\partial\left(u_{1}^{\left(\beta_{1}\right)}, \cdots, u_{m}^{\left(\beta_{m}\right)}\right)}\right] \neq 0 .
$$

A large class of nonlinear systems, especially mechanical systems, are naturally in this form. Additionally, a wider class of nonlinear systems, termed as 'differentially flat systems', can be written in this form together with dynamic compensators which may be a chain of integrators in their simplest sense (Fliess (1988)).

The system (7) is called minimum phase if the zero dynamics, $\phi_{i}(0, \hat{u}, t)=0 ; i=1, \cdots, p$ are uniformly asymptotically stable. Only proper minimum phase systems are considered in this paper.

The system (7) can be written in the following generalised canonical form

$$
\begin{aligned}
\dot{\xi}_{1}^{i} & =\xi_{2}^{i} \\
& \ldots \\
\dot{\xi}_{n_{i}-1}^{i} & =\xi_{n_{1}}^{i} \\
\dot{\xi}_{n_{i}}^{i} & =\phi_{i}(\xi, \hat{u}, t), \quad i=1, \cdots, m
\end{aligned}
$$

where $\xi^{i}=\left(\xi_{1}^{i}, \cdots, \xi_{n_{i}}^{i}\right)=\left(y_{i}, \cdots, y_{i}^{\left(n_{i}-1\right)}\right), i=$ $1, \cdots, m$ and $\xi=\left(\xi^{1}, \cdots, \xi^{m}\right)$.

The sliding surface, $s$, is selected such that when it is made zero, the control objective is achieved. In the case of MIMO systems, the sliding surface is a vector which has the same dimension as 
that of the control vector $u$ i.e., $s \in \Re^{m}$. In general, the sliding surface can be selected as a nonlinear function of the system states such as $s=\Psi(\xi, t)$. However, only linear sliding surfaces are considered here as follows

$$
s_{i}=\xi_{n_{i}}^{i}+\sum_{j=1}^{n_{i}-1} c_{j}^{i} \xi_{j}^{i}, \quad i=1, \cdots, m
$$

where the vector of constants $\left(c_{1}^{i}, c_{2}^{i}, \cdots, c_{n_{i}-1}^{i}\right)$ are such that the polynomials

$$
\lambda_{i}^{n_{i}}+\sum_{j=1}^{n_{i}-1} c_{j}^{i} \lambda_{i}^{j}=0 ; \quad i=1, \cdots, m
$$

are Hurwitz.

It should be noted that the sliding surface in equation (9) is naturally control dependent if $\beta_{i} \geq 1$ for $i=1, \cdots, m$. However, as will be seen in this section, for a dynamic controller to result as is required for chattering removal, no condition on $\beta$ is imposed here. This is contrary to what is proposed by Sira-Ramirez (1993) where $\beta \geq 1$ is required for the controller to be dynamic, and thus for chattering avoidance. Moreover, the definition (9) does not assume that the sliding surface is control dependent. Therefore, the methodology is different from what is proposed by Lu and Spurgeon (1998), where the sliding surface is always control dependent. The first two successive time derivatives of the sliding variables (9) along the system trajectories are given by

$$
\begin{aligned}
\dot{s}_{i}= & \phi_{i}(\xi, \hat{u}, t)+c_{n_{i}-1}^{i} \xi_{n_{i}}^{i}+\sum_{j=1}^{n_{i}-2} c_{j}^{i} \xi_{j+1}^{i} \\
\ddot{s}_{i}= & \frac{\partial \phi_{i}}{\partial t}+\sum_{k=1}^{m} \sum_{j=1}^{n_{k}} \frac{\partial \phi_{i}}{\partial \xi_{j}^{k}} \xi_{j+1}^{k}+\sum_{k=1}^{m} \sum_{j=0}^{\beta_{k}-1} \frac{\partial \phi_{i}}{\partial u_{k}^{(j)}} u_{k}^{(j+1)} \\
& +c_{n_{i}-1}^{i} \phi_{i}(\xi, \hat{u}, t) \\
& +c_{n_{i}-2}^{i} \xi_{n_{i}}^{i}+\sum_{j=1}^{n_{i}-3} c_{j}^{i} \xi_{j+2}^{i}+\sum_{k=1}^{m} \frac{\partial \phi_{i}}{\partial u_{k}^{\left(\beta_{k}\right)}} u_{k}^{\left(\beta_{k}+1\right)} \\
& \quad i=1, \cdots, m
\end{aligned}
$$

Let $z^{i}=\left(z_{1}^{i}, z_{2}^{i}, \cdots, z_{\beta_{i}}^{i}\right)=\left(u_{i}, \dot{u}_{i}, \cdots, u_{i}^{\left(\beta_{i}\right)}\right), z=$ $\left(z^{1}, \cdots, z^{m}\right)$ and $u_{i}^{\left(\beta_{i}+1\right)}=v_{i}$ for $i=1, \cdots, m$.

The control $u$ can be implemented using a chain of integrators with auxiliary input $v_{i}$ which can be written in Brunovsky canonical form as

$$
\dot{z}^{i}=G_{i} z^{i}+H_{i} v_{i}, \quad i=1, \cdots, m
$$

Using equation (9), the system (8) can be written as

$$
\begin{aligned}
& \dot{\hat{\xi}}^{i}=A_{i} \hat{\xi^{i}}+B_{i} s_{i} \\
& \ddot{s}_{i}=f_{i}(\xi, z, t)+g_{i}(\xi, z, t) v_{i} \\
& \dot{z}^{i}=G_{i} z^{i}+H_{i} v_{i}, \quad i=1, \cdots, m
\end{aligned}
$$

where $\hat{\xi}^{i}=\left(\xi_{1}^{i}, \cdots, \xi_{n_{i}-1}^{i}\right)$ and

$$
\begin{aligned}
f_{i}(\xi, z, t)= & \frac{\partial \phi_{i}}{\partial t}+\sum_{k=1}^{m} \sum_{j=1}^{n_{k}} \frac{\partial \phi_{i}}{\partial \xi_{j}^{k}} \xi_{j+1}^{k}+ \\
& \sum_{k=1}^{m} \sum_{j=0}^{\beta_{k}-1} \frac{\partial \phi_{i}}{\partial u_{k}^{(j)}} u_{k}^{(j+1)}+c_{n_{i}-1}^{i} \phi_{i}(\xi, \hat{u}, t) \\
& +c_{n_{i}-2}^{i} \xi_{n_{i}}^{i}+\sum_{j=1}^{n_{i}-3} c_{j}^{i} \xi_{j+2}^{i} \\
g_{i}(\xi, z, t)= & {\left[\frac{\partial \phi_{i}}{\partial u_{1}^{\left(\beta_{1}\right)}} u_{1}^{\left(\beta_{1}+1\right)}, \cdots, \frac{\partial \phi_{i}}{\partial u_{m}^{\left(\beta_{m}\right)}} u_{m}^{\left(\beta_{m}+1\right)}\right] }
\end{aligned}
$$

and

$$
\begin{aligned}
& A_{i}=\left[\begin{array}{ll}
0 & I_{n-2} \\
& -C
\end{array}\right], \quad B_{i}=\left[\begin{array}{l}
0 \\
1
\end{array}\right] \\
& G_{i}=\left[\begin{array}{cc}
0 & I_{\beta_{i}-1} \\
& 0
\end{array}\right], \quad H_{i}=\left[\begin{array}{l}
0 \\
1
\end{array}\right]
\end{aligned}
$$

The complete MIMO system thus can be written as

$$
\begin{aligned}
& \dot{\hat{\xi}}=A \hat{\xi}+B s \\
& \ddot{s}=f(\xi, z, t)+g(\xi, z, t) v \\
& \dot{z}=G z+H v
\end{aligned}
$$

where $\hat{\xi}=\left(\hat{\xi}^{1}, \cdots, \hat{\xi}^{m}\right) \in \Re^{n-m}, s=\left(s_{1}, \cdots, s_{m}\right) \in$ $\Re^{m}$ and $v=\left(v_{1}, \cdots, v_{m}\right) \in \Re^{m}$ and $A=$ $\operatorname{diag}\left(A_{1}, \cdots, A_{m}\right), B=\operatorname{diag}\left(B_{1}, \cdots, B_{m}\right), G=$ $\operatorname{diag}\left(G_{1}, \cdots, G_{m}\right), H=\operatorname{diag}\left(H_{1}, \cdots, H_{m}\right), f=$ $\left(f_{1}, \cdots, f_{m}\right)^{T}, g=\left(g_{1}, \cdots, g_{m}\right)^{T} \in \Re^{m \times m}$.

In this paper, only those system types are considered where the input gain matrix $g$ is diagonal, i.e.,

$$
g=\operatorname{diag}\left(g_{i i}\right), \text { where } g_{i i}(\xi, z, t)=\frac{\partial \phi_{i}}{\partial u_{i}^{\left(\beta_{i}\right)}}
$$

Therefore, the original MIMO system produces three subsystems. The first subsystem is an $(n-$ $m$ ) dimensional linear system driven by the sliding variable vector, $s$. This linear subsystem is stable as the polynomials in (9) are Hurwitz by design. The second subsystem represents the second order nonlinear uncertain dynamics of the $m$ sliding variables. If bounding values of $f_{i}$ and $g_{i i}$ (for $i=1, \cdots, m$ ) satisfy the conditions (3), the algorithm (4)-(5) can be applied for robust stabilisation of each channel independently. The third subsystem is a chain of integrators, the output of which provides the actual control. If the system is linearizable by coordinate change and state feedback, the MIMO system representation in I-O form (7) will be independent of any control derivative, i.e.,

$$
\beta_{j}=0, \quad j=1, \cdots, m
$$

and therefore, the auxiliary control vector, $v$, which drives the sliding surface dynamics (16) is 
simply the time derivative of the actual control vector $u$. Even though $v$ is discontinuous, the actual control input, $u$, to the system will be smooth because of the integrator dynamics.

Once the sliding surface dynamics in (16) is stabilised to $s=0$, the MIMO system dynamics under sliding motion is given by

$$
\dot{\hat{\xi}}=A \hat{\xi}
$$

which is stable because $A$ is a block diagonal matrix with all diagonal blocks $\left(A_{i}, i=1, \cdots, m\right)$ representing the stable dynamics (10).

Though the system in (16) is coupled with the states of the system in (15), it can be decoupled if the vector field $f(\xi, z, t)$ and the gain matrix $g(\xi, z, t)$, even if uncertain, satisfy the following bounding conditions in any bounded domain

$$
\begin{gathered}
\left|f_{i}(\xi, z, t)\right|<F_{i} \\
G_{m_{i}} \leq g_{i i}(\xi, z, t) \leq G_{M_{i}}
\end{gathered}
$$

and the system can be written as

$$
\begin{aligned}
& \dot{y}_{1_{i}}=y_{2_{i}} \\
& \dot{y}_{2_{i}}=f_{i}(\xi, z, t)+g_{i i}(\xi, z, t) v_{i}
\end{aligned}
$$

This representation contains all those uncertainties which do not violate the bounding conditions (19). The algorithm (4) can be applied because the control $v_{i}$ always appears linearly. The controller parameters are selected as follows

$$
\begin{gathered}
u_{0_{i}}>\sup F_{i} / G_{m_{i}} \\
\lambda_{i}>u_{0_{i}}, k_{i}>0, W_{i}>0
\end{gathered}
$$

Sometimes, the controller (21) may be conservative. To improve the conservatism, the conditions (19) imposed on the sliding surface dynamics can be relaxed as

$$
\begin{gathered}
\left|f_{i}(\xi, z, t)\right|<F_{i_{1}}+F_{i_{2}}\left|y_{1_{i}}\right| \\
G_{m_{1 i}}+G_{m_{2 i}}\left|y_{1_{i}}\right| \leq g_{i i}(\xi, z, t) \leq G_{M_{1 i}}+G_{M_{2 i}} \mid y_{1_{i}}(22)
\end{gathered}
$$

and the controller parameters can be selected as follows

$$
\begin{gathered}
u_{0_{i}}>\frac{F_{i_{1}}+F_{i_{2}}\left|y_{1_{i}}\right|}{G_{m_{1 i}}+G_{m_{2}}\left|y_{1_{i}}\right|} \\
\lambda_{i}>u_{0_{i}}, k_{i}>0, W_{i}>0
\end{gathered}
$$

\section{LIQUID LEVEL CONTROL IN COUPLED-TANKS}

The twin-tanks system consists of two small tanks mounted above a reservoir which provides storage for the water. Water is pumped into the bottom of each tank by two independent pumps. The pump only increases the liquid level and is not responsible for pumping the water out of the tank. It is assumed that the back pressure created by the water-head does not affect the flow rate of the pump significantly. The separating wall between the two tanks has two circular holes which together form the connecting pipe with admittance coefficient $k_{1}$; this will be referred to as pipe $k_{1}$. Each tank in the twin tanks configuration is equipped with two outlet pipes of different radius. These two outlet pipes have admittance coefficients of $k_{2}$ and $k_{3}$ where $k_{3}>k_{2}$ and will be referred to as pipe $k_{2}$ and pipe $k_{3}$, respectively. The admittance coefficients of various pipes are assumed constant. The pump cannot fill the tank if both pipes $k_{2}$ and $k_{3}$ are opened simultaneously. Therefore, only pipe $k_{2}$ is considered open for designing the controller. A leak is simulated by opening pipe $k_{3}$ (and pipe $k_{2}$ closed) which allows more outflow than pipe $k_{2}$. The schematic diagram of the system is shown in Fig. 1.

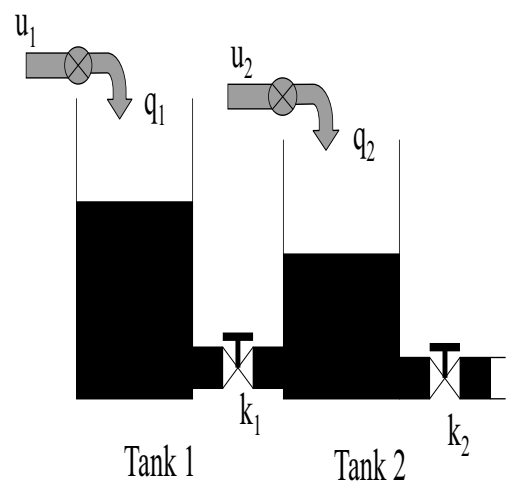

Fig. 1. The liquid-level control system

The twin-connected tanks system is a nonlinear dynamical system and the governing dynamical equations can be written as (Chang (1990))

$$
\begin{aligned}
& \dot{h}_{1}=-\frac{k_{1}}{A_{1}} \sqrt{\left|h_{1}-h_{2}\right|} \operatorname{sign}\left(h_{1}-h_{2}\right)+\frac{1}{A_{1}} q_{1} \\
& \dot{h}_{2}=\frac{k_{1}}{A_{2}} \sqrt{\left|h_{1}-h_{2}\right|} \operatorname{sign}\left(h_{1}-h_{2}\right)-\frac{k_{2}}{A_{2}} \sqrt{h_{2}}+\frac{1}{A_{2}} q_{2}
\end{aligned}
$$

where $h_{1}$ and $h_{2}$ are the total water heads in Tank 1 and Tank 2 respectively, which are the two outputs of interest and $q_{1}, q_{2}$, are the two inflows into the tanks. It is assumed that the capacities, $A_{1}$ and $A_{2}$, of Tank-1 and Tank-2 respectively remain bounded as follows

$$
A_{i_{m}} \leq A_{i} \leq A_{i_{M}}, \quad i=1,2 .
$$

where $A_{i_{m}}$ and $A_{i_{M}}$ are some positive constants. For the rig in the laboratory both tanks have the same cross-sectional area i.e. $A_{1}=A_{2}=A$.

It has been seen in the work of Kantor (1989) and Clark and Vinter (2003) that in chemical plants, selecting the flow rate as an input is more 
effective than using flow as the input. Thus, if the flow rates are considered as the inputs, i.e. $\dot{q}_{1}=u_{1}$ and $\dot{q}_{2}=u_{2}$, the system dynamics can be written in $\mathrm{I}-\mathrm{O}$ form as

$$
\begin{aligned}
& \ddot{h}_{1}=f_{1}\left(h_{1}, h_{2}, \dot{h}_{1}, \dot{h}_{2}\right)+\frac{1}{A} u_{1} \\
& \ddot{h}_{2}=f_{2}\left(h_{1}, h_{2}, \dot{h}_{1}, \dot{h}_{2}\right)+\frac{1}{A} u_{2}
\end{aligned}
$$

where

$$
\begin{aligned}
& f_{1}(\cdot)=-\frac{k_{1}}{2 A} \frac{\dot{h}_{1}-\dot{h}_{2}}{\sqrt{\left|h_{1}-h_{2}\right|}} \\
& f_{2}(\cdot)=\frac{k_{1}}{2 A} \frac{\dot{h}_{1}-\dot{h}_{2}}{\sqrt{\left|h_{1}-h_{2}\right|}}-\frac{k_{2}}{2 \sqrt{h_{2}}} \dot{h}_{2}
\end{aligned}
$$

It seems from equations (24) and (25) that there is a singularity in the trajectories at $h_{1}=h_{2}$. In fact, this is not so because at $h_{1}=h_{2}$, the system model is decoupled. The fluid flow into the tanks ( $q_{1}$ and $q_{2}$ ) cannot be negative because the pumps can only pump water into the tanks. Therefore constraints on the inflow are given by

$$
q_{1} \geq 0
$$

and

$$
q_{2} \geq 0
$$

In the steady state, for constant water level set points, the respective derivatives must be zero separately i.e., $\dot{h}_{1}=\dot{h}_{2}=0$. Therefore, in the steady state, the steady state inflows $\left(Q_{1}, Q_{2}\right)$ are given by

$$
\begin{aligned}
& Q_{1}=k_{1} \sqrt{\left|h_{1}-h_{2}\right|} \operatorname{sign}\left(h_{1}-h_{2}\right) \\
& Q_{2}=-k_{1} \sqrt{\left|h_{1}-h_{2}\right|} \operatorname{sign}\left(h_{1}-h_{2}\right)+k_{2} \sqrt{h_{2}}
\end{aligned}
$$

Using equation (29), the constraint (27) on the input can be reformulated as a constraint on the output set point as follows

$$
\sqrt{\left|h_{1}-h_{2}\right|} \operatorname{sign}\left(h_{1}-h_{2}\right) \geq 0 \Rightarrow h_{1} \geq h_{2}
$$

Similarly, constraint (28) can be reformulated using (30) as follows

$$
\begin{aligned}
k_{2} \sqrt{h_{2}} & \geq k_{1} \sqrt{\left|h_{1}-h_{2}\right|} \operatorname{sign}\left(h_{1}-h_{2}\right) \\
\left(k_{1}^{2}+k_{2}^{2}\right) h_{2} & \geq k_{1}^{2} h_{1} \\
\Rightarrow h_{2} & \geq \frac{k_{1}^{2}}{k_{1}^{2}+k_{2}^{2}} h_{1}
\end{aligned}
$$

Therefore, in order to satisfy the constraints (27) and (28) on the inflows for given values of the plant parameters $k_{1}, k_{2}$, the desired liquid levels in the tanks must satisfy the constraints (31) and (32) which can be combined as follows

$$
\frac{k_{1}^{2}}{k_{1}^{2}+k_{2}^{2}} \leq \frac{h_{2}}{h_{1}} \leq 1
$$

For the plant available in laboratory, the water level in Tank $2, h_{2}$, must not be theoretically less than $63.89 \%$ that of $h_{1}$ to satisfy positive flow constraints.

For the laboratory rig, both tanks have the same area of $155.44 \mathrm{~cm}^{2}$ and $k_{1}=23.45$ and $k_{2}=17.62$. The set point water level is selected as $h_{1_{d}}=$ $h_{2_{d}}=12 \mathrm{~cm}$. For the given plant, bounding values for the functions $A f_{1}(\cdot)$ and $A f_{2}(\cdot)$ are calculated by maximising all the possible variations as $A\left|f_{1}(\cdot)\right|_{\max }=23.4$ and $A\left|f_{2}(\cdot)\right|_{\max }=33.7$.

\section{CONTROLLER DEVELOPMENT}

For both the tanks, the error in the water level is selected as the sliding surface i.e.,

$$
s_{i}=h_{i}-h_{i_{d}} ; \quad i=1,2 .
$$

The sliding surface variable, $s_{i}$, in equation (34) has relative degree two with respect to the control, $v_{i}$. The complete MIMO system according to the equations (15-17) can be written as

$$
\begin{aligned}
& \ddot{s}_{i}=f_{i}\left(h_{i}, \dot{h}_{i}\right)+g_{i}\left(h_{i}, \dot{h}_{i}\right) v_{i} \\
& \dot{u}_{i}=v_{i} \quad i=1,2 .
\end{aligned}
$$

where $f_{1}(\cdot)$ and $f_{2}(\cdot)$ are according to equation (26) and $g_{1}(\cdot)=g_{2}(\cdot)=1 / A$. The control algorithm (4) is then applied to stabilise the decoupled dynamics (35).

The controller parameters $u_{0_{1}}$ and $u_{0_{2}}$ should be greater than $A\left|f_{1}(\cdot)\right|_{\max }$ and $A\left|f_{2}(\cdot)\right|_{\max }$ respectively. The controller parameters selected for the simulation study shown in the following figures are $u_{0_{1,2}}=[25,35], \lambda_{1,2}=[100,150], k_{1,2}=[1,1]$ and $W_{1,2}=[10,10]$, which satisfy the selection criteria discussed in the previous section. Using equations (29) and (30), the inflows at equilibrium are $Q_{1}=0$ and $Q_{2}=61.07$.

The implementation results are shown in the following figures. Note that a digital output of 150 corresponds to a liquid level of $12 \mathrm{~cm}$. It has been noticed that the pumps do not respond to digital inputs less that 25 in the case of Tank 1 and 30 in the case of Tank 2. To counter this dead zone, a lower saturation limit of 25 and 30 is applied to both motor inputs respectively. The controller parameters selected are $\lambda=[150,200]$, $u_{0}=[75,100], k=[37,37]$ and $W=[300,300]$.

To simulate a leak in Tank 1 , the outlet pipe with admittance coefficient equal to $k_{2}$ is opened. In the case of Tank 2, a leak condition has been simulated by opening the outlet pipe with wider cross sectional area (admittance coefficient $k_{3}=$ 26.26) than that of the pipe which has been used to model the system $\left(k_{1}=23.45\right)$. The controller robustly stabilises the water levels to the desired level in both cases. 

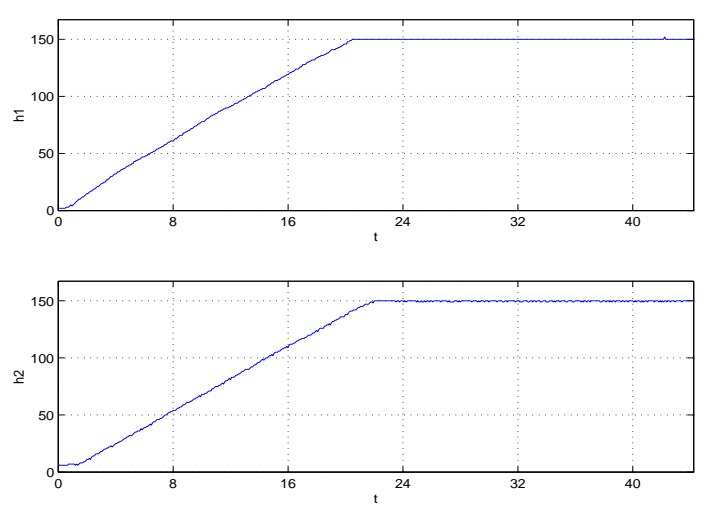

Fig. 2. Stabilisation to the same water level (150 units) in both tanks
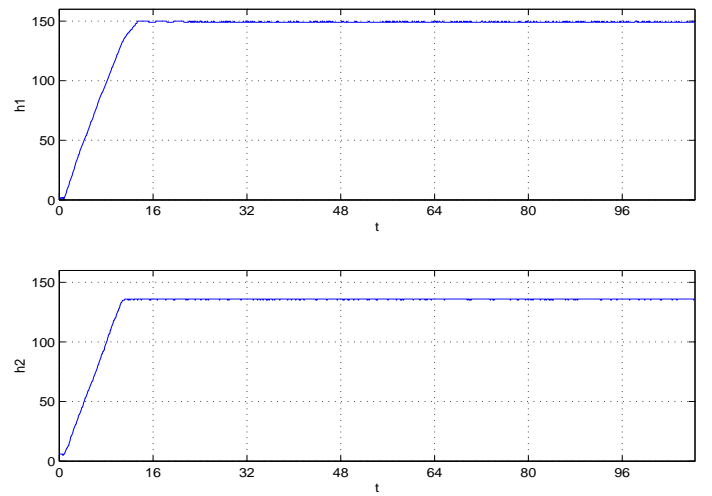

Fig. 3. Stabilisation to different water levels (150 and 135 units) in both tanks
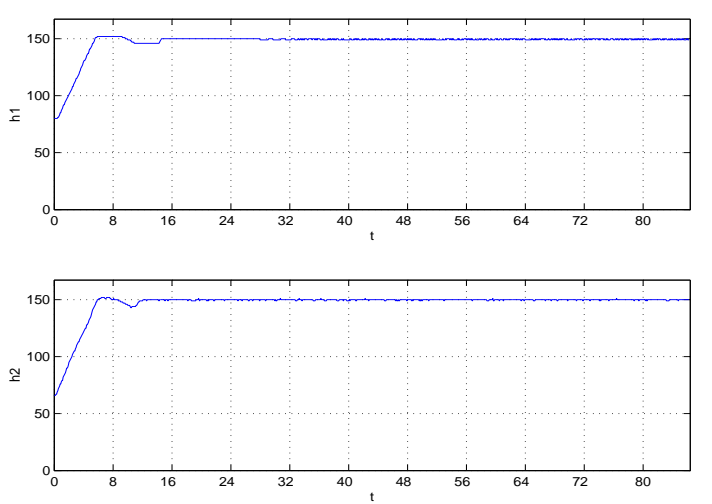

Fig. 4. Stabilisation with a leak in tank 1

\section{CONCLUSIONS}

A second order sliding mode control algorithm has been presented for robust control of the liquid level in a pair of interconnected tanks. The algorithm does not require the derivative of the sliding surfaces, thus eliminating the requirement of designing an observer or peak detector, contrary to many other sliding mode control strategies. The system has been modelled and implementation of the controller has been carried out on an experimental rig. The implementation results show robustness to parameter variations such as tank area and the admittance coefficients of various pipes. The implementation results verify the potential of second order sliding modes for control of MIMO systems.

\section{REFERENCES}

Bartolini, G., Ferrara, A., Usai, E., Utkin, V., 2000. On multi-input chattering-free-second order sliding mode control. IEEE Trans. on Automatic Control 45(9), 1711-1718.

Chang, L., 1990. A MIMO sliding control with a second order sliding condition. In: ASME Winter Annual Meeting. Dallas, Texas, paper No. 90-WA/DSC-5.

Clark, J., Vinter, R., 2003. A differential dynamic games approach to flow control. In: $42^{\text {nd }}$ IEEE Conference on Decision and Control. Hawaii, USA.

Edwards, C., Spurgeon, S., 1998. Sliding mode control: Theory and applications. Taylor \& Francis.

Fliess, M., 1988. Nonlinear control theory and differential algebra. In: Byrnes, C., Kurzhanski, A. (Eds.), Modelling and adaptive control. Vol. 105 of Lecture Notes in Control and Information Sciences. Springer-Verlag, New York, pp. 134145.

Fliess, M., 1990. Generalized controller canonical form for linear and nonlinear dynamics. IEEE Trans. on Automatic Control 35(9), 994-1001.

Kantor, J., 1989. Non-linear sliding mode controller and objective function for surge tanks. Int. J. Control 50, 2025-2047.

Khan, M., Spurgeon, S., Levant, A., 2003. Simple output feedback 2-sliding controller for systems of relative degree two. In: European Control Conference (ECC). Cambridge, UK.

Lu, X., Spurgeon, S., 1998. Asymptotic stabilization of multiple input nonlinear systems via sliding modes. Dynamics and Control 8, 231254.

Lu, X., Spurgeon, S., 1999. Output feedback stabilization of MIMO nonlinear systems via dynamic sliding modes. Int. J. Robust Nonlinear Control 9, 275-305.

Sira-Ramirez, H., 1993. On the dynamic sliding mode control of nonlinear systems. Int. J. Control 57(5), 1039-1061.

Utkin, V., 1992. Sliding modes in control and optimization. Springer-Verlag. 